\title{
Notas corológicas para la provincia de Sevilla
}

\author{
Santiago Martín Bravo1, José Manuel Herrera² \& Iñigo Pulgar \\ 'Área de Botánica, Departamento de Biología Molecular e Ingeniería Bioquímica, Universidad Pablo de Olavide, Ctra. \\ de Utrera Km 1, 41013, Sevilla \\ ${ }^{2}$ C/Sor María Trillo, 15, 41620, Marchena (Sevilla)
}

\author{
Correspondencia \\ S. Martín Bravo \\ e-mail: smarbra@upo.es \\ Recibido: 1 octubre 2018 \\ Aceptado: 11 septiembre 2019 \\ Publicado on-line: 26 septiembre 2019 \\ Editado por: B. Cabezudo
}

\author{
Chorological notes for Seville province \\ Palabras clave: Corología, angiospermas, novedades, Andalucía, Sevilla.
}

Key words: Chorology, angiosperms, new records, Andalusia, Seville.
La realización de estudios florísticos en la provincia de Sevilla ha dado lugar a algunas novedades corológicas de interés para la flora de Andalucía occidental, que detallamos a continuación. La mayor parte de ellas corresponden a novedades para la comarca natural de Los Alcores (11 taxones), aunque también para otras comarcas (Campiña Alta sevillana, 3 taxones; Condado-Aljarafe, 2 taxones) definidas en Flora vascular de Andalucía occidental (Valdés et al., 1987), así como al hallazgo de nuevas poblaciones para 2 especies amenazadas. Materiales testigo de cada una de las especies comentadas han sido depositados en el herbario de la Universidad Pablo de Olavide de Sevilla (UPOS). Para la identificación y estudio de la distribución de las especies se utilizaron como referencia las obras Flora vascular de Andalucía occidental (Valdés et al., 1987), Flora vascular de Andalucía oriental (Blanca et al., 2011) y Flora iberica (Castroviejo et al., 1986-2018).

Ammoides pusilla (Brot.) Breistr. (Apiaceae)

SEVILLA. Alcalá de Guadaíra, Monumento Natural Riberas del Guadaíra, cerro de los Ángeles, eucaliptar sobre calcarenitas, 6/6/2014, A. Gálvez Montes 207AGM14 (UPOS 10771).

Elemento mediterráneo citado de la mayoría de las comarcas del centro y oeste de Andalucía occidental (García Martín, 1987); más recientemente ha sido citada también para la Campiña de Huelva (Sánchez Gullón, 1999). Completamos su conocimiento corológico en Andalucía occidental con esta cita que constituye novedad comarcal para los Alcores.
Filago lusitanica (Samp.) P. Silva (Asteraceae)

SEVILLA. Alcalá de Guadaíra, finca de Piedrahincada, palmitar-retamar-encinar sobre suelos calcareníticos, 22/3/2013, A. Mestre Jiménez 193AMJ13 (UPOS 9683).

El género Filago L. presenta gran complejidad taxonómica y su sistemática ha sufrido notables modificaciones en losúltimos años, comolainclusión de las especies que tradicionalmente constituían el género Evax Gaertn. (Andrés-Sánchez et al., 2013). Una de ellas es este endemismo iberomagrebí conocido hasta el momento del norte y oeste de Andalucía Occidental, donde crece en campos incultos sobre suelos ácidos (Devesa, 1987). Novedad para la comarca de Los Alcores, donde las características rocas calcareníticas aparecen con frecuencia meteorizadas originando suelos de textura arenosa donde se instalan especies de apetencias más acidófilas como esta (Silvestre Alsina et al., 2013).

Hedysarum glomeratum F. Dietr. (Fabaceae)

SEVILLA. Camas, Paraje La Atalaya, cerro de Santa Brígida, laderas sobre suelos margosos básicos, exposición sur, c. $95 \mathrm{~m}, 4 / 4 / 2017, \mathrm{~S}$. Martín Bravo 20SMB17 (UPOS 8940).

Leguminosa de distribución mediterránea con una remarcable disyunciónen su distribuciónibérica, entre el extremo nororiental y el suroccidental de la Península (Valdés, 2000). Inicialmente conocida del sur de Andalucía occidental (Domínguez, 1987), ha sido encontrada más recientemente en la Subbética cordobesa (Triano, 1998), Campiña Baja sevillana (Roales, 1998), y Sierra Norte sevillana 
(Valdés, 2005); la presente cita es novedad para la comarca de Condado-Aljarafe.

Juncus inflexus L. subsp. inflexus (Juncaceae)

SEVILLA. Alcalá de Guadaíra, Monumento Natural Riberas del Guadaíra, cerro de los Ángeles, riberas del río Guadaíra, 15 m, 6/6/2014, A. Gálvez Montes 177AGM14 (UPOS 9685). La Puebla de Cazalla, Las Juntas, borde de arroyo sobre arcillas, 30STG9612, 234 m, 13/04/2016, J.M. Herrera \& I. Pulgar (UPOS 8285).

Táxon ampliamente distribuido en las regiones templadas del Viejo Mundo y también en la Península lbérica, donde habita en praderas y juncales húmedos frecuentemente nitrificados (Romero Zarco, 2010a). Primeras citas para la comarca de Los Alcores y Campiña Alta sevillana (Fernández-Carvajal, 1987); ha sido recientemente citado de la comarca limítrofe de la Vega (Romero Zarco, 2010b).

\section{Lens lamottei Czefr. (Fabaceae)}

SEVILLA. Alcalá de Guadaíra, Monumento Natural Riberas del Guadaíra, cerro de los Ángeles, laderas en eucaliptar sobre suelos básicos (calcarenitas), c. 40 m, 10/5/2018, S. Martín Bravo \& P. Escobar 43SMB18 (UPOS 10688).

Especie mediterránea pobremente conocida cuya distribución es incierta, puesto que ha sido frecuentemente confundida con la estrechamente emparentada L. nigricans (M. Bieb.) Godr. (Castroviejo \& Pascual, 1995). Esta última era la única especie recogida en el tratamiento de Flora de Andalucía occidental (Romero Zarco, 1987; véase también Galiano \& Valdés, 1976). En la Península Ibérica $L$. lamottei ha sido indicada de la franja costera mediterránea y sur de Portugal (Castroviejo \& Pascual, 1999). La presente cita, novedad para la comarca de Los Alcores, se encuentra más alejada del litoral de lo que cabría esperar (Castroviejo \& Pascual, 1999), y constituye un nuevo ejemplo de disyunción en especies calcícolas entre Los Alcores y las poblaciones previamente conocidas de las sierras subbéticas calizas (Castroviejo \& Pascual, 1995; Silvestre Alsina et al., 2013). A pesar de las dudas sobre el estatus nativo de esta especie en Europa (ILDIS, 2010), su distribución y hábitat en la península nos inclinan a pensar que es autóctona (Castroviejo \& Pascual, 1995, 1999).

\section{Lonicera implexa Aiton (Caprifoliaceae)}

SEVILLA. Carmona, finca La Ranilla, lentiscaracebuchar con Quercus coccifera, Cistus albidus y Jasminum fruticans sobre suelos pobres básicos, 22/3/2017, S. Martín Bravo 14SMB17, E. Peña \&
F. Rodríguez, (UPOS 8957).

Novedad comarcal encontrada en el extremo norte de Los Alcores, en su límite con la Vega del Guadalquivir (valle del río Corbones).

\section{Ophrys bombyliflora Link (Orchidaceae)}

SEVILLA. Camas, Paraje La Atalaya, cerro de Santa Brígida, ladera con acebuches sobre suelos margosos básicos, exposición norte, c. $100 \mathrm{~m}$, 4/4/2017, S. Martín Bravo 23SMB17 (UPOS 8942).

Orquídea mediterráneo-macaronésica distribuida en la mitad sur de la Península Ibérica (Aldasoro \& Saéz, 2005). Está considerada como bastante rara en Andalucía, tanto en su parte oriental como occidental (Silvestre, 1987a; Algarra \& Blanca, 2011), aunque resulta localmente frecuente en puntos de la Campiña Baja gaditana y Algeciras (Sánchez García \& Martínez Ortega, 1994). Completamos su conocimiento corológico en Andalucía con esta primera cita para la comarca de Condado-Aljarafe.

Osyris lanceolata Hochst. \& Steud. (Santalaceae) SEVILLA. Carmona, finca El Judío, cantil calcarenítico xerófilo con acebuche y lentisco, c. 215 m, 22/3/2017, S. Martín Bravo 5SMB17, E. Peña \& F. Rodríguez. (UPOS 8948).

Arbusto ibero-magrebí que alcanza Baleares y Canarias. En la Península Ibérica, está distribuido en la mitad sur, donde crece generalmente en matorrales mediterráneos litorales o sublitorales (Aedo, 1997). En Andalucía occidental, se pensaba restringido a arenales costeros de Huelva y Cádiz (Ruiz de Clavijo, 1987a), pero posteriores contribuciones corológicas han ampliado considerablemente su distribución a Sierra Morena y Subbética occidental incluyendo las provincias de Huelva, Sevilla, Córdoba, Jaén y Badajoz (Albarreal \& Romero Zarco, 1993; Rodríguez Marzal \& Pérez-Carra, 2009). La población aquí indicada constituye la primera localidad para la comarca natural de los Alcores, que aparece como reducto disyunto entre las poblaciones serranas y costeras de esta especie. Estos hallazgos también ponen de manifiesto la amplitud de sus preferencias edáficas, pues se conocen poblaciones sobre dunas estabilizadas, suelos cuarcíticos, y roquedos graníticos y calizos.

\section{Prasium majus L. (Lamiaceae)}

SEVILLA. Montellano, Monumento Natural Tajos de Mogarejo, matorral termomediterráneo, zonas umbrías sobre bloques calizos del puente, 105 m, 17/6/2018, S. Martín Bravo 67SMB18 (UPOS 10689).

Novedad para la comarca natural de la 
Campiña Alta de este elemento mediterráneomacaronésico que en España está distribuido en el extremo sur de la Península Ibérica, Mallorca y Canarias (Morales, 2010a).

\section{Proboscidea louisianica (Mill.) Thell. \\ (Martyniaceae) \\ SEVILLA. La Puebla de Cazalla, embalse} de la Puebla de Cazalla, cerca de la orilla, sobre margas, 30SUG0011, 250 m, 16/8/2018, J.M. Herrera \& I. Pulgar (UPOS 10762).

Especie originaria de Méjico y $\mathrm{S}$ de los Estados Unidos que en la Península Ibérica se naturaliza en el $\mathrm{C}$ y mitad $\mathrm{W}$ en zonas baldías y ruderales algo nitrificadas (Paiva, 2001). En Andalucía la presencia de este taxón es conocida desde fechas relativamente recientes, y tan sólo en su mitad occidental, en las comarcas Sierra Norte sevillana (Moglia et al., 2001) y Zújar (Muñoz-Álvarez et al., 2016). Primera cita para la Campiña Alta.

\section{Salix alba L. (Salicaceae)}

SEVILLA. Alcalá de Guadaíra, Monumento Natural riberas del Guadaíra, cerro de los Ángeles, riberas del río Guadaíra, 6/6/2014, A. Gálvez Montes 173AGM14 (UPOS 9680).

Árbol de ribera de distribución paléartica disperso en la Península Ibérica. En Andalucía oriental, Ilama la atención la aparente contradicción entre el tratamiento de Flora iberica que lo cita de todas las provincias (Blanco, 2005), y el más reciente de Flora de Andalucía oriental, que lo indica como muy raro y presente solo en los tramos altos de ríos y arroyos de las sierras de Cazorla (Salazar \& Quesada, 2011). En Andalucía occidental, su presencia en la comarca de Los Alcores había pasado desapercibida hasta el momento (Silvestre, 1987b; García Montoya \& Muñoz, 1990; Sánchez García \& Martínez Ortega, 1994; Morales et al., 2000; Romero Zarco, 2004).

\section{Sideritis arborescens Salzm. ex Benth.} (Lamiaceae)

SEVILLA. Carmona, cueva de la Batida, 2012, E. Peña Pérez 48EPP12 (UPOS 10559). Los Palacios, camino de la N-IV a la Hacienda de Seixa, 40-50 m, suelos arenosos al borde del camino, 2/6/2005, S. Martín Bravo 315SMB05 (UPOS 5998).

Endemismo ibero-magrebí que en la Península es exclusivo de las provincias litorales del sur (Morales, 2010b). Los especímenes aquí citados constituyen novedades comarcales para Los Alcores y la Vega del Guadalquivir. No obstante, ya se conocía la presencia de $S$. $x$ gaditana $(S$. arborescens x S. hirsuta) en Los Alcores (Socorro,
1987; Silvestre Alsina et al., 2013).

\section{Silene mariana Pau (Caryophyllaceae)}

SEVILLA. Dos Hermanas, cerros junto al Hipódromo, olivares semi-abandonados y acebuchares con Genista hirsuta, sobre coluviones aluviales, c. 70 m, 8/5/2018, S. Martín Bravo 35SMB18 (UPOS 10693). Alcalá de Guadaíra, Monumento Natural riberas del Guadaíra, cerro de los Ángeles, laderas rocosas sobre afloramientos calcareníticos, c. 40 m. 10/5/2018, S. Martín Bravo \& P. Escobar (UPOS 10690).

Endemismo exclusivo de la mitad sur peninsular (Talavera, 1990) e incluido en la Lista roja de la flora vascular española (Moreno, 2010) y la Lista roja de la flora vascular de Andalucía (Cabezudo et al., 2005) con la categoría de "Casi Amenazada" (NT). Además cuenta con protección legal a nivel nacional al estar recogida en el Listado de Especies Silvestres en Régimen de Protección Especialdesarrollado por el Real Decreto 139/2011, así como a nivel andaluz, a raíz de su inclusión en el Listado Andaluz de Especies Silvestres en Régimen de Protección Especial. En Andalucía occidental está distribuida disyuntamente entre Sierra Morena cordobesa, Sierra de Grazalema y Los Alcores, comarca esta última en la que se ubican las dos nuevas poblaciones, en ambos casos en zonas de contacto con la Vega del Guadalquivir (Talavera, 1987a). Parece existir una cierta controversia sobre los requerimientos ecológicos de esta especie, para la que se ha indicado afinidad por sustratos preferentemente calizos (Talavera, 1987a, 1990) o ácidos (Díaz de la Guardia, 2011). Las dos poblaciones encontradas crecen sobre sustratos claramente distintos, lo que parece sugerir la indiferencia edáfica de esta especie. En la población de Dos Hermanas y en una de las poblaciones previamente conocidas de Los Alcores en Alcalá de Guadaíra se han producido recientemente incendios que parecen haberla favorecido. Llamamos la atención especialmente sobre las potenciales amenazas a la población de Dos Hermanas, pues se encuentra en una zona entre grandes núcleos de población (Sevilla, Dos Hermanas y Montequinto) que está actualmente sometida a una intensa actividad de expansión urbanística.

Soliva stolonifera (Brot.) Sweet (Asteraceae)

SEVILLA. Carmona, finca La Ranilla, lentiscaracebuchar con Quercus coccifera, Cistus albidus y Jasminum fruticans sobre suelos pobres básicos, 22/3/2017, S. Martín Bravo 16SMB17, E. Peña \& F. Rodríguez (UPOS 8959).

Esta compuesta es originaria de América 
del Sur y hasta el momento sólo se conoce su naturalización en Europa en la Península Ibérica y Macaronesia (Greuter, 2006). Suele aparecer en medios más antrópicos que los de esta población, como fisuras de aceras y calzadas (Talavera, 1987b); la presente cita es la primera registrada en la comarca de Los Alcores. Su distribución parece ir en aumento en Andalucía occidental, a la vista de contribuciones corológicas previas (Jiménez \& Ruíz de Clavijo, 1990; Pinilla et al., 1998; López Tirado et al., 2013), aunque parece aún muy rara en Andalucía oriental (Blanca, 2011).

Trachelium caeruleum L. subsp. caeruleum (Campanulaceae)

SEVILLA. Alcalá de Guadaíra, Monumento Natural Riberas del Guadaíra, Molino de la Aceña, paredones calcareníticos rezumantes, $30 \mathrm{~m}$, 11/12/2016, S. Martín Bravo 46SMB16 \& L. Bellón (UPOS 9059).

Taxón íbero-magrebí de distribución dispersa en la Península Ibérica (Sales \& Hedge, 2001). En Andalucía occidental, aunque se conocía de todas las provincias (Sales \& Hedge, 2001), no estaba registrada para el valle del Guadalquivir (Cabezudo, 1987). Esta población, que presenta una ecología típica, constituye una novedad para la comarca de Los Alcores, la cual parece cumplir para este táxon el papel de isla edáfica en la depresión del Guadalquivir ya propuesto para otras especies calcícolas en Andalucía occidental (Silvestre Alsina et al., 2013).

\section{Triguera osbeckii (L.) Willk. (Solanaceae)}

SEVILLA. Camas, Paraje La Atalaya, cerca del campo de tiro, pastizales en laderas sobre suelos básicos, c. 60 m, 7/3/2017, S. Martín Bravo 3SMB17 (UPOS s.n.)

Endemismo íbero-magrebí catalogado como especie Vulnerable en las listas rojas nacional (Moreno, 2010) y andaluza (Cabezudo et al. 2005), aunque no cuenta con protección legal. Su distribución en Andalucía occidental ha sido recientemente revisada por Jiménez-Mejías et al. (2014). Hacemos mención a esta nueva población en la comarca de Condado-Aljarafe, a pesar de su cercanía a otras citas anteriores (ver JiménezMejías et al., 2014), por su condición de especie amenazada.

\section{Viscum cruciatum Boiss. (Loranthaceae)}

SEVILLA. Carmona, finca El Judío, sobre acebuches, en laderas rocosas calizas con orientación sureste, c. 180 m, 22/3/2017, S. Martín Bravo 11SMB17, E. Peña \& F. Rodríguez (UPOS 8955).
Especie íbero-magrebí y asiática que en la Península Ibérica es exclusiva del suroeste (Catalán \& Aparicio, 1997). En Andalucía occidental, está considerada como poco frecuente en las comarcas de Condado-Aljarafe (aunque está dada por extinta en la provincia de Huelva; Catalán \& Aparicio, 1997), Campiña Baja gaditana, Subbética sevillana, Grazalema (Ruiz de Clavijo, $1987 b)$ y Campiña Alta cordobesa (García Montoya \& Muñoz, 1990). Parasita diversas dicotiledóneas leñosas, entre las que se encuentra Olea europaea L. La presente cita es novedad comarcal (Alcores) y destaca por su baja altitud, ya que esta especie se encuentra habitualmente entre 300 y 1600 m (Catalán \& Aparicio, 1997; Gómez Mercado, 2011).

\section{Agradecimientos}

A A. Mestre Jiménez y A. Gálvez Montes por sus recolecciones en el término municipal de Alcalá de Guadaíra y a E. Peña y F. Rodríguez en el de Carmona. A P. Jiménez-Mejías y R. Morales por su ayuda en la determinación de algunos taxones. Al personal del herbario UPOS, especialmente a José Manuel Cobos por su apoyo técnico.

\section{Bibliografía}

Aedo, C. (1997). Osyris L. In G. Nieto Feliner \& C. Aedo (Eds.). Flora iberica 8 (pp. 149-152). Madrid: CSIC.

Albarreal, J. \& Romero Zarco, C. (1993). Novedades florísticas para la campiña y las sierras subbéticas sevillanas. Lagascalia 17(1), 190-193.

Aldasoro, J.J. \& Saéz, L. (2005). Ophrys L. In C. Aedo \& A. Herrero (Eds.). Flora Iberica 21 (pp. 165-195). Madrid: CSIC.

Algarra, J., \& Blanca, G. (2011). Ophrys L. In G. Blanca, B. Cabezudo, M. Cueto, C. Salazar \& C. Morales Torres (Eds.). Flora Vascular de Andalucía Oriental (pp. 221-227). Granada: Universidades de Almería, Granada, Jaén y Málaga.

Andrés-Sánchez, M., Martínez-Ortega, M. \& Rico, E. (2013). Estudio corológico del género Filago L. (Asteraceae, Gnaphalieae) en la Península Ibérica y Baleares. Botanica Complutensis 37: 57-78.

Blanca, G. (2011). Soliva Ruiz \& Pav. In G. Blanca, B. Cabezudo, M. Cueto, C. Salazar \& C. Morales Torres (Eds.). Flora Vascular de Andalucía Oriental (pp. 1633). Granada: Universidades de Almería, Granada, Jaén y Málaga.

Blanca, G., Cabezudo, B., Cueto, M., Salazar, C. \& Morales Torres, C. (Eds.) (2011). Flora Vascular de Andalucía Oriental. Granada: Universidades de Almería, Granada, Jaén y Málaga.

Blanco, P. (2005). Salix L. In C. Soriano (Ed.). Flora Iberica 3 (pp. 477-517). Madrid: CSIC.

Cabezudo, B. (1987). Trachelium L. In B. Valdés, S. Talavera \& E.F. Galiano (Eds.). Flora vascular de Andalucía occidental 2 (pp. 567). Barcelona: Ketres. 
Cabezudo, B., Talavera, S., Blanca, G., Salazar, C., Cueto, M., Valdés, B., Hernández Bermejo, J.E., Herrera, C.M., Rodríguez Hiraldo, C. \& Navas, D. (2005). Lista roja de la flora vascular de Andalucía. Sevilla: Consejería de Medio Ambiente, Junta de Andalucía.

Castroviejo, S. (coord. gen.) (1986-2018). Flora iberica. Madrid: CSIC.

Castroviejo, S. \& Pascual, H. (1995). Notas sobre el género Lens Mill. (Leguminosae) en la Península Ibérica e Islas Baleares. Anales del Jardín Botánico de Madrid 53(2), 177-180.

Castroviejo, S. \& Pascual, H. (1999). Lens Mill. In S. Talavera, C. Aedo, S. Castroviejo, C. Romero, L. Sáez, F.J. Salgueiro \& M. Velayos (Eds.). Flora iberica 7(1) (pp. 417-423). Madrid: CSIC.

Catalán, P. \& Aparicio, A. (1997). Viscum L. In G. Nieto Feliner (Ed.). Flora iberica 8 (pp. 160-164). Madrid: CSIC.

Devesa, J.A. (1987) Evax Gaertn. In B. Valdés, S. Talavera \& E.F. Galiano (Eds.). Flora vascular de Andalucía occidental 3 (pp. 28-30). Barcelona: Ketres.

Díaz de la Guardia, C. (2011). Silene L. In G. Blanca, B. Cabezudo, M. Cueto, C. Salazar \& C. Morales Torres (Eds.). Flora Vascular de Andalucía Oriental (pp. 520537). Granada: Universidades de Almería, Granada, Jaén y Málaga.

Domínguez, E. (1987). Hedysarum L. In B. Valdés, S. Talavera \& E.F. Galiano (Eds.). Flora Vascular de Andalucía occidental 2 (pp. 86-88). Barcelona: Ketres.

Fernández-Carvajal, M.C. (1987). Juncus L. In B. Valdés, S. Talavera \& E.F. Galiano (Eds.). Flora vascular de Andalucía occidental 3 (pp. 213-228). Barcelona: Ketres.

Galiano, E.F. \& Valdés, B. (1976) Catálogo de las Plantas Vasculares de la Provincia de Sevilla. VIII. (Papilionaceae). Lagascalia 6, 38-89.

García Martín, F. (1987). Ammoides Adans. In B. Valdés, S. Talavera \& E.F. Galiano (Eds.). Flora vascular de Andalucía occidental 2 (pp. 318). Barcelona: Ketres.

García Montoya, F. \& Muñoz, M.J. (1990). Novedades corológicas para la Flora de Andalucía Occidental. Lagascalia 16(1), 146-168.

Gómez Mercado, F. (2011). Viscum L. In G. Blanca, B. Cabezudo, M. Cueto, C. Salazar \& C. Morales Torres (Eds.). Flora Vascular de Andalucía Oriental (pp. 627628). Granada: Universidades de Almería, Granada, Jaén y Málaga.

Greuter, W. (2006+) Compositae (pro parte majore). In W. Greuter \& E. von Raab-Straube (Ed.). Compositae. Euro+Med Plantbase - the information resource for Euro-Mediterranean plant diversity. http://www. emplantbase.org/, accedido en julio de 2018.

ILDIS (2010). World Database of Legumes. https://ildis. org/index.shtml, accedido en septiembre de 2018

Jiménez, M. \& Ruíz de Clavijo, E. (1990). Nuevas áreas para la Flora de Andalucía Occidental. Lagascalia 16, 132-145.

Jiménez Mejías, P., Peña Pérez, E., Rodríguez León, F. \& Martín Bravo, S. (2014). Triguera osbeckii (L.) Willk. (Solanaceae) redescubierta en Carmona (Comarca de los Alcores, Sevilla). Acta Botanica Malacitana 39:
239-241.

López Tirado, J., Muñoz Álvarez, J.M. \& Hidalgo Fernández, P.J. (2013). Aportaciones a la flora vascular de la provincia de Córdoba I (Andalucía, España). Lagascalia 33, 314-325.

Moglia, M.M., Delgado Marzo, J.M., Sánchez Almendro, A. \& Muñoz Álvarez, J.M. (2001). Flora de interés corológico para Andalucía occidental. Acta Botanica Malacitana, 26, 260-262.

Morales, M., Tamajón, R., Delgado, J.M. \& Muñoz, J.M. (2000). Novedades corológicas para la flora de la provincia de Huelva (comarcas de Aracena y Sierra Norte). Lagascalia 21, 351-364.

Morales, R. (2010a). Prasium L. In R. Morales \& A. Quintanar (Eds.). Flora iberica 12 (pp. 232-234). Madrid: CSIC.

Morales, R. (2010b). Sideritis L. In R. Morales \& A. Quintanar (Eds.). Flora iberica 12 (pp. 234-288). Madrid: CSIC.

Moreno, J.C., Coord. (2010). Lista Roja de la Flora Vascular Española 2008, actualización con los datos del Adenda 2010 al Atlas y Libro Rojo de la Flora Vascular Amenazada. Madrid: Dirección General de Conservación de la Naturaleza y Sociedad Española de Biología de la Conservación de Plantas.

Muñoz-Álvarez, J.M., Delgado-Marzo, J.M. \& DíazIglesias, M.L. (2016). Aportaciones a la flora alóctona de Córdoba y Andalucía occidental (España). Botanica Complutensis 40, 135-145.

Pavia, J. (2001). Proboscidea Schmidel. In J. Pavia, A. Herrero \& C. Aedo (Eds.). Flora Iberica 14 (pp. 22-24). Madrid: CSIC.

Pinilla, R., Tamajón, R. \& Muñoz, J.M. (1998). Aportaciones a la flora de Córdoba. Acta Botanica Malacitana 23, 260-269.

Roales, J. (1998). Contribución al conocimiento de la flora de Sevilla. II: novedades corológicas en el Valle del Guadalquivir. Lagascalia 20(2): 239-256.

Rodríguez Marzal, J.L. \& Pérez-Carral, C. (2009). Algunas plantas de interés de la Sierra de Aguafría (Monesterio, Badajoz). Botanica Complutensis 33, 45-51.

Romero Zarco, C. (1987). Lens Mill. In B. Valdés, S. Talavera \& E.F. Galiano (Eds.). Flora vascular de Andalucía occidental 2 (pp. 63). Barcelona: Ketres.

Romero Zarco, C. (2004). Sobre algunos neófitos y otras citas interesantes para la flora de Andalucía Occidental. Acta Botanica Malacitana, 29, 305-310.

Romero Zarco, C. (2010a). Juncus L. In C. Romero Zarco, S. Talavera \& A. Herrero (Eds.) Flora iberica 17 (pp. 123-187). Madrid: CSIC.

Romero Zarco, C. (2010b). El género Juncus L. (Juncaceae) en Andalucía (España): datos sobre la distribución regional de sus especies. Acta Botanica Malacitana 35, 57-75.

Ruíz de Clavijo, E. (1987a). Osyris L. In B. Valdés, S. Talavera \& E.F. Galiano (Eds.). Flora vascular de Andalucía occidental 2 (pp. 211-212). Barcelona: Ketres.

Ruíz de Clavijo, E. (1987b). Viscum L. In B. Valdés, S. Talavera \& E.F. Galiano (Eds.). Flora vascular de Andalucía occidental 2 (pp. 214). Barcelona: Ketres.

Sales, F. \& Hedge, I.C. (2001). Trachelium L. In F. Sales, 
I.C. Hedge, S. Castroviejo \& J.J. Aldasoro (Eds.). Flora iberica 14 (pp. 141-142). Madrid: CSIC.

Salazar, C. \& Quesada, J. (2011). Salix L. In G. Blanca, B. Cabezudo, M. Cueto, C. Salazar \& C. Morales Torres (Eds.). Flora Vascular de Andalucía Oriental (pp. 703-710). Granada: Universidades de Almería, Granada, Jaén y Málaga.

Sánchez García, I. \& Martínez Ortega, C. (1994). Nuevas áreas para la Flora de Andalucía Occidental. Lagascalia 17(2), 357-366.

Sanchéz Gullón, E. (1999). Novedades corológicas para la provincia de Huelva. Acta Botanica Malacitana 24, 242-247.

Silvestre, S. (1987a). Ophrys L. In B. Valdés, S. Talavera \& E.F. Galiano (Eds.). Flora vascular de Andalucía occidental 3 (pp. 513-519). Barcelona: Ketres.

Silvestre, S. (1987b). Salix L. In B. Valdés, S. Talavera \& E.F. Galiano (Eds.). Flora vascular de Andalucía occidental 1 (pp. 367-371). Barcelona: Ketres.

Silvestre Alsina, A., Martín Bravo, S. \& Jiménez Mejías, P. (2013). Catálogo de la flora vascular del Cerro del Toruño (Comarca de Los Alcores, Alcalá de Guadaira, Sevilla). Lagascalia 33, 7-18.

Socorro, O. (1987). Sideritis L. In B. Valdés, S. Talavera
\& E.F. Galiano (Eds.). Flora vascular de Andalucía occidental 2 (pp. 428-433). Barcelona: Ketres.

Talavera, S. (1987a). Silene L. In B. Valdés, S. Talavera \& E.F. Galiano (Eds.). Flora vascular de Andalucía occidental 1 (pp. 244-267). Barcelona: Ketres.

Talavera, S. (1987b). Gymnostyles Juss. In B. Valdés, S. Talavera \& E.F. Galiano (Eds.). Flora vascular de Andalucía occidental 3 (pp. 69). Barcelona: Ketres.

Talavera, S. (1990). Silene L. In M. Laínz \& F. Muñoz Garmendía (Eds.). Flora iberica 2 (pp. 313-406). Madrid: CSIC.

Triano, E.C. (1998). Flora del Subbético Cordobés. Rute (Córdoba): Ayuntamiento de Rute. Excma. Diputación Provincial de Córdoba.

Valdés, B. (2000). Hedysarum L. In Talavera, S., C. Aedo, S. Castroviejo, C. Romero, L. Sáez, F.J. Salgueiro \& M. Velayos (Eds.). Flora iberica 7(2) (pp. 943-955). Madrid: CSIC.

Valdés, B. (2005). Nuevas plantas y nuevas áreas para la flora de Andalucía y $\mathrm{N}$ de Marruecos. I. Lagascalia 25, 193-204.

Valdés, B., Talavera, S. \& Fernández-Galiano, E. (1987). Flora Vascular de Andalucía Occidental. Barcelona: Ketres. 